\title{
Ginsenoside Rb1 protects the intestinal mucosal barrier following peritoneal air exposure
}

\author{
FENG ZHOU $^{1}$, PEICHEN ZHANG ${ }^{1}$, XIAOXI CHEN ${ }^{1}$, JINGYI YAN ${ }^{1}$, \\ JIANGAO YAO ${ }^{1}$, ZHEN YU ${ }^{1,2}$ and XIAOLEI CHEN ${ }^{1}$ \\ ${ }^{1}$ Department of Gastrointestinal Surgery, The First Affiliated Hospital of Wenzhou Medical University, \\ Wenzhou, Zhejiang 325000; ${ }^{2}$ Department of General Surgery, Shanghai Tenth People's Hospital Affiliated to Tongji University, \\ Shanghai 200072, P.R. China
}

Received June 16, 2015; Accepted June 15, 2016

DOI: $10.3892 / \mathrm{etm} .2016 .3639$

\begin{abstract}
Ginsenoside Rb1 (GRb1), which is one of the main ingredients derived from Panax ginseng, has been widely used to treat various gastrointestinal disorders. The present study aimed to determine whether GRb1 was able to prevent intestinal mucosal barrier damage in rats following peritoneal air exposure for $3 \mathrm{~h}$. GRb1 $(5,10$, and $20 \mathrm{mg} / \mathrm{kg}$ ) was orally administrated via gavage four times prior to and following surgery. Blood and terminal ileum were sampled $24 \mathrm{~h}$ following surgery. Levels of serum D-lactate (D-LA) were detected using an enzyme-linked immunosorbent assay kit. Intestinal permeability was assessed by determining the intestinal clearance of fluorescein isothiocyanate-dextran (FD4). Activity of intestinal myeloperoxidase was measured to assess intestinal inflammation, and intestinal histopathology was assessed by light microscopy. The results showed that GRb1 reduced the level of serum D-LA, intestinal clearance of FD4, and the activity of intestinal myeloperoxidase. Intestinal edema and inflammation were also ameliorated by GRb1, and the Chiu's scores employed for assessing intestinal mucosal damage were also reduced in the GRb1-treated peritoneal air exposure group. In addition, GRb1 induced a significant difference at 10 and $20 \mathrm{mg} / \mathrm{kg}$, indicating a dose-dependent effect. The results of the present study suggest that GRb1 may be able to protect the intestinal mucosal barrier against damage induced by peritoneal air exposure, which may be associated with its anti-inflammatory action.
\end{abstract}

Correspondence to: Dr Xiaolei Chen or Dr Zhen Yu, Department of Gastrointestinal Surgery, The First Affiliated Hospital of Wenzhou Medical University, 2 Fuxue Lane, Wenzhou, Zhejiang 325000, P.R. China

E-mail: chenxiaoleiwy@163.com

E-mail: yuzhentongji@163.com

Key words: ginsenoside Rb1, intestinal mucosal barrier, peritoneal cavity

\section{Introduction}

Ginseng, which is the dried root of Panax ginseng C.A. Meyer (Araliaceae) (1), has been well accepted as a drug to treat various gastrointestinal diseases in traditional Chinese medicine. Ginsenosides are the main active ingredients of ginseng and have many pharmacological actions, including anti-tumor, anti-oxidant, anti-inflammation and anti-fatigue effects (2-5). Ginsenoside Rb1 (GRb1) is one of the main ginsenosides. Recent research has demonstrated that GRb1 is able to maintain intestinal epithelial tight junctions (6), improving the functional performance of the intestinal mucosal barrier in a number of diseases, including intestinal ischemia-reperfusion injury and intestinal microflora disorders $(7,8)$.

In modern surgery, it is important to ensure the patient's efficient recovery; however, various factors in the perioperative period may affect postoperative rehabiliation. In open abdominal surgery, peritoneal air exposure is very common, which can lead to tissue or organ injury (9-11). Furthermore, the gut is easily injured by physical stress and is thus often considered as the cause of multiple organ dysfunction in critical illness (12). A recent study demonstrated that peritoneal air exposure may cause intestinal mucosal barrier dysfunction, which is proportionally associated with the duration of peritoneal air exposure (13). However, intestinal bacteria and endotoxins may be translocated to remote organs when the intestinal mucosal barrier is damaged, and this intestinal mucosal barrier dysfunction may further result in inflammation of the intestine or other organs (14). In clinical practice, various complications are derived from intestinal injury and inflammation following open abdominal surgery (15-17). Therefore, effective methods to improve intestinal mucosal barrier function in modern surgery are required.

In the present study, the potential role of GRb1 in preventing intestinal mucosal barrier damage caused by peritoneal air exposure was explored, in experimental conditions simulating those of a surgical environment in clinical practice.

\section{Materials and methods}

Experimental animals. Healthy adult male Sprague-Dawley rats $(6.5 \pm 0.3$ weeks; $210-230 \mathrm{~g})$ were purchased from Shanghai 
SLAC Laboratory Animal Co., Ltd., (Shanghai, China). Rats were housed in the laboratory under controlled temperature $\left(20-22^{\circ} \mathrm{C}\right)$, humidity $(45-55 \%)$ and light $(12 \mathrm{~h} \mathrm{light/dark} \mathrm{cycle)}$ conditions, and had ad libitum access to standard rat chow and tap water. Experimental procedures involving the rats were checked and approved by the Institutional Animal Committee of Wenzhou Medical University (Wenzhou, China). Rats were treated in accordance with the guidelines of the National Institutes of Health (Bethesda, MD, USA).

Reagents. GRb1 (purity, >98\%) was purchased from Shanghai Tauto Biotech Co., Ltd., (Shanghai, China). All chemicals and reagents used in the present study were of analytical grade.

Animal grouping and reagent administration. Following an adaptation period of one week, 40 rats were randomly divided into five groups $(\mathrm{n}=8)$ : Control group $(\mathrm{CG})$; peritoneal air exposure for $3 \mathrm{~h}$ group (PG); and three groups that received GRb1 following PG treatment at doses of 5, 10 and $20 \mathrm{mg} / \mathrm{kg}$, respectively (GG, including GG5, GG10 and GG20). Rat models of peritoneal air exposure for $3 \mathrm{~h}$ were established according to a previous study (13). Briefly, following anesthesia with $2 \%$ pentobarbital sodium (Sigma-Aldrich; Merck Millipore, Darmstadt, Germany) at a dose of $3.5 \mathrm{ml} / \mathrm{kg}$, rats in the PG and GG groups were laparotomized with a $3 \mathrm{~cm}$ midline incision, followed by peritoneal air exposure for $3 \mathrm{~h}$. Surgery was performed in a sterile environment with controlled humidity and temperature. Rats in the CG group were exposed to the same anesthesia as the PG and GG groups, without surgery. Rats in the $\mathrm{CG}$ and $\mathrm{PG}$ groups received saline by gavage once daily at 3,2 and 1 days prior to surgery, and at $6 \mathrm{~h}$ post-surgery. Rats in the GG groups were administered GRb1 dissolved in saline at a dose of 5,10 and $20 \mathrm{mg} / \mathrm{kg}$ in the respective groups four times via the same method as the CG group (4). At $24 \mathrm{~h}$ post-surgery, blood and terminal ileum were harvested to provide samples for subsequent detection. Terminal ileum was harvested at the detected site, according to previous studies (18-20), which determined that the terminal ileum was the optimal site for the investigation of intestinal mucosal barrier damage following exposure to various types of injury. This indicates that the most sensitive section in the gut is located at the terminal ileum.

Serum D-lactate (D-LA) measurement. Inferior vena cava was chosen as the harvesting site for blood samples. Blood was centrifuged at $800 \mathrm{x} \mathrm{g}$ for $15 \mathrm{~min}$ at $4^{\circ} \mathrm{C}$ to collect serum. Serum D-LA levels were measured using an ELISA kit for rats (cat. no. 10597R-09; R\&D Systems, Inc., Minneapolis, MN, USA) according to the manufacturer's protocol.

Intestinal permeability measurements. Intestinal clearance of fluorescein-isothiocyanate dextran (FD4) was measured to assess intestinal permeability according to previously published studies $(13,18,19)$. Briefly, an $8-\mathrm{cm}$ section of terminal ileum was removed, and the mucosa was gently everted. One end of this gut segment was closed with a 4-0 silk suture and $1.0 \mathrm{ml}$ Krebs-Henseleit bicarbonate buffer was injected from the opposite end to prepare an intestinal sac. This filled intestinal sac was subsequently incubated in a solution containing $0.5 \mathrm{mg} / \mathrm{ml} \mathrm{FD4} \mathrm{(average} \mathrm{molecular} \mathrm{weight:}$
$4,000)$ at $37^{\circ} \mathrm{C}$. Bathing solution was gently aerated and bubbled with a gas mixture containing $5 \% \mathrm{CO}_{2}$ and $95 \% \mathrm{O}_{2}$ for $30 \mathrm{~min}$, and the mucosal surface area value was subsequently determined. Solution fluorescence was detected spectrophotometrically, and intestinal FD4 clearance was measured using the following formula:

$$
\mathrm{C}=\frac{[\mathrm{FD} 4]_{\mathrm{ser}} \times 1 \mathrm{ml}}{[\mathrm{FD} 4]_{\mathrm{muc}} \times \mathrm{A} \times 30 \mathrm{~min}} \mathrm{~A}=\pi L D
$$

In this formula, $\mathrm{C}$ indicates the mucosal to serosal clearance of FD4 in $\mu 1 \cdot \mathrm{min}^{-1} \cdot \mathrm{cm}^{-2}$; A denotes the mucosal surface area; $[\text { FD } 4]_{\text {muc }}$ is the concentration of FD4 in the mucosal solution aspirated from the sac at the beginning of the $30 \mathrm{~min}$ period; [FD4 $]_{\text {ser }}$ is the concentration of FD4 in the serosal solution aspirated from the sac at the end of the 30 min period; $L$ denotes the sac length; and $D$ indicates the sac diameter.

Intestinal myeloperoxidase (MPO) activity determination. Tissue of the terminal ileum was homogenized and centrifuged at $2,000 \mathrm{xg}$ for $15 \mathrm{~min}$ at $4^{\circ} \mathrm{C}$ to obtain the supernatants. The concentration of supernatant protein was detected by the Bradford method (21). MPO activity was quantitatively assessed using spectrophotometry at $460 \mathrm{~nm}$ as, reported in a previous study (11). MPO activity was reported as units/g in the gut tissue.

Histopathological observation. Terminal ileum was prepared and fixed in $4 \%$ buffered formaldehyde, and embedded with paraffin. Slices (4- $\mu \mathrm{m}$ thick) were cut, stained using hematoxylin and eosin, and observed by a pathologist using light microscopy. The pathologist was blinded to the research design. Degree of gut mucosa damage was assessed by Chiu's scoring system, as reported in a previous study (22). Alterations in the gut mucosa following air exposure to the peritoneal cavity were graded as follows: Grade 0, normal gut mucosal villi with no injury; grade 1, sub-epithelial Gruenhagen's space was typically developed at the top of the gut villus, and often presented capillary congestion; grade 2, sub-epithelial Gruenhagen's space was extended and the epithelial layer was moderately lifted from the lamina propria; grade 3, massive gut epithelial lifting down the sides of gut villi, some were denuded; grade 4, villi were denuded from the lamina propria, dilated capillaries were exposed and the cellularity of the lamina propria was increased; and grade 5, the lamina propria was digested and disintegrated with both ulceration and hemorrhage in gut mucosa.

Statistical analysis. All data were presented as mean \pm standard deviation. Statistical analysis was performed using a SPSS software package for Windows (version 17.0; SPSS, Inc., Chicago, IL, USA). Data were analyzed via one-way analysis of variance, and the least significant difference test was used for multi-group comparisons. $\mathrm{P}<0.05$ was considered to indicate a statistically significant difference.

\section{Results}

Effects of GRbl on the serum level of D-LA. Compared with the $\mathrm{CG}$ group, the $\mathrm{PG}$ group exhibited a significantly increased serum level of D-LA $(\mathrm{P}<0.05$; Fig. 1). However, the three GG 


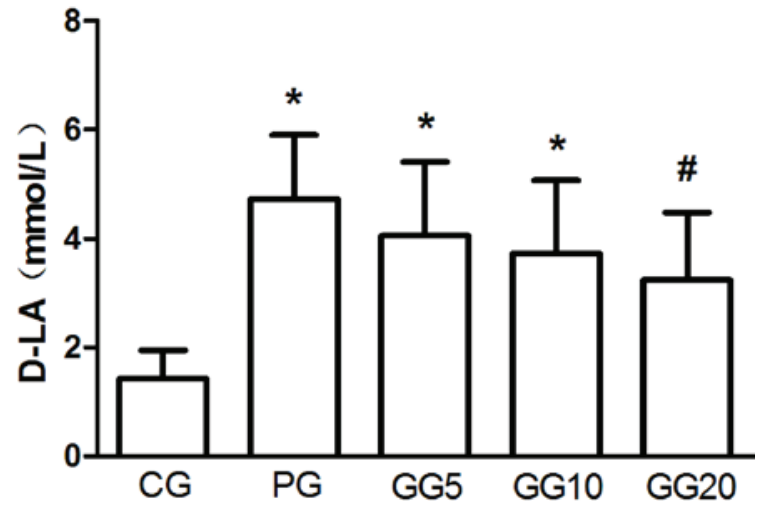

Figure 1. Effects of GRb1 on the serum level of D-LA. Results are presented as mean \pm standard deviation. ${ }^{*} \mathrm{P}<0.05$, vs. $\mathrm{CG}$, ${ }^{\#} \mathrm{P}<0.05$, vs. $\mathrm{PG}$. GRb1, ginsenoside Rb1; D-LA, D-lactate, CG, control group; PG, peritoneal air exposure for $3 \mathrm{~h}$ group; GG5, $5 \mathrm{mg} / \mathrm{kg}$ GRb1-treated PG group; GG10, $10 \mathrm{mg} / \mathrm{kg}$ GRb1-treated PG group; GG20, 20 mg/kg GRb1-treated PG group.

groups demonstrated a progressively decreasing serum D-LA level, in a dose-dependent manner. This decrease was significant in the GG20 group when compared with the PG group $(\mathrm{P}<0.05)$. Furthermore, although the GG5 and GG10 groups demonstrated significantly increased serum levels of D-LA when compared with the CG group $(\mathrm{P}<0.05)$, no significant differences in the serum level of D-LA were detected between the GG20 and CG groups.

Effects of GRb1 on intestinal permeability. Compared with the CG group, the $\mathrm{PG}$ group demonstrated a significant increase in intestinal FD4 clearance ( $\mathrm{P}<0.05$; Fig. 2). However, the GG groups demonstrated a progressively decreasing intestinal FD4 clearance in a dose-dependent manner. This decrease was significant in the GG20 group when compared with the PG group $(P<0.05)$, although the GG20 group still exhibited significantly increased intestinal FD4 clearance than that of the $\mathrm{CG}$ group $(\mathrm{P}<0.05)$.

Effects of GRb1 on the activity of intestinal MPO. Compared with the CG group, the PG group showed significantly increased activity of intestinal MPO ( $\mathrm{P}<0.05$; Fig. 3). However, the three GG groups demonstrated progressively decreasing MPO activity in a dose-dependent manner, with significance detected in the GG10 and the GG20 groups when compared with the PG group $(\mathrm{P}<0.05)$. Furthermore, although the GG5 and GG10 groups demonstrated significantly increased MPO activity when compared with the CG group $(\mathrm{P}<0.05)$, no significant difference was observed between the MPO activity of the GG20 and CG groups.

Histopathological observation. No marked structural changes were observed in the gut tissue samples among the five groups (Fig. 4); however, compared with the CG group, the PG group demonstrated edema and inflammation in the gut mucosa and submucosa. Administration of GRb1 ameliorated this edema and inflammation in the three GG groups when compared with those in the PG group. Furthermore, as demonstrated in Fig. 5, the GG groups also exhibited progressively decreasing Chiu's scores in a dose-dependent manner, with significance detected between the GG20 and PG groups $(\mathrm{P}<0.05)$.

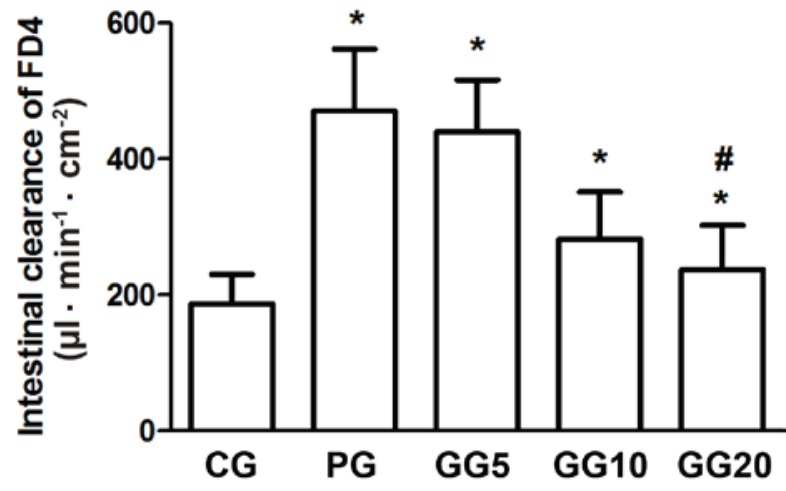

Figure 2. Effects of GRb1 on intestinal FD4 clearance. Results are presented as mean \pm standard deviation. ${ }^{*} \mathrm{P}<0.05$, vs. $\mathrm{CG},{ }^{~} \mathrm{P}<0.05$, vs. $\mathrm{PG}$. GRb1, ginsenoside Rb1; FD4, fluorescein isothiocyanate-dextran; CG, control group; PG, peritoneal air exposure for $3 \mathrm{~h}$ group; GG5, $5 \mathrm{mg} / \mathrm{kg}$ GRb1-treated PG group; GG10, 10 mg/kg GRb1-treated PG group; GG20, 20 mg/kg GRb1-treated PG group.

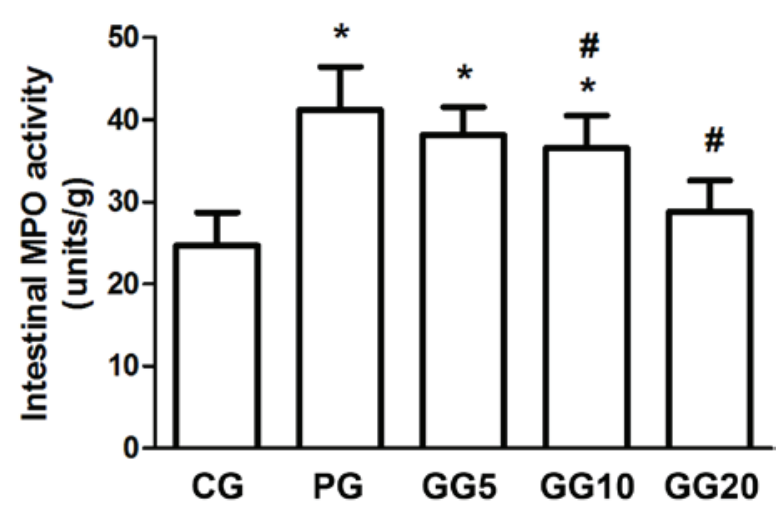

Figure 3. Effects of GRb1 on the activity of intestinal MPO. Results are presented as mean \pm standard deviation. ${ }^{*} \mathrm{P}<0.05$, vs. CG, ${ }^{\#} \mathrm{P}<0.05$, vs. PG. GRb1, ginsenoside Rb1; MPO, myeloperoxidase; $\mathrm{CG}$, control group; $\mathrm{PG}$, peritoneal air exposure for $3 \mathrm{~h}$ group; GG5, $5 \mathrm{mg} / \mathrm{kg}$ GRb1-treated PG group; GG10, 10 mg/kg GRb1-treated PG group; GG20, 20 mg/kg GRb1-treated PG group.

\section{Discussion}

In the present study, the prevention of intestinal mucosal barrier from damage caused by peritoneal air exposure by GRb1 administration was investigated. The results demonstrated that GRb1 decreased serum levels of D-LA, intestinal clearance of FD4, and the activity of intestinal MPO. Intestinal edema and inflammation were ameliorated by GRb1, and Chiu's score and signs of gut mucosal injury were decreased in the GRb1 treated groups. Furthermore, this protective effect exerted by GRb1 occurred in a dose-dependent manner, with significant differences detected at doses of 10 and $20 \mathrm{mg} / \mathrm{kg}$.

Until recently, it has been difficult to assess the functionality of the intestinal mucosal barrier. Typically, intestinal mucosal barrier function is assessed indirectly by measuring intestinal permeability, via the measurement of serum D-LA levels in various bacterial infection diseases (23-25). Therefore, D-LA is often chosen as a biomarker to assess intestinal mucosal barrier function.

FD4 is a relatively large molecule that is unable to pass through the intestinal mucosal barrier under normal conditions. However, intestinal permeability may be increased in 

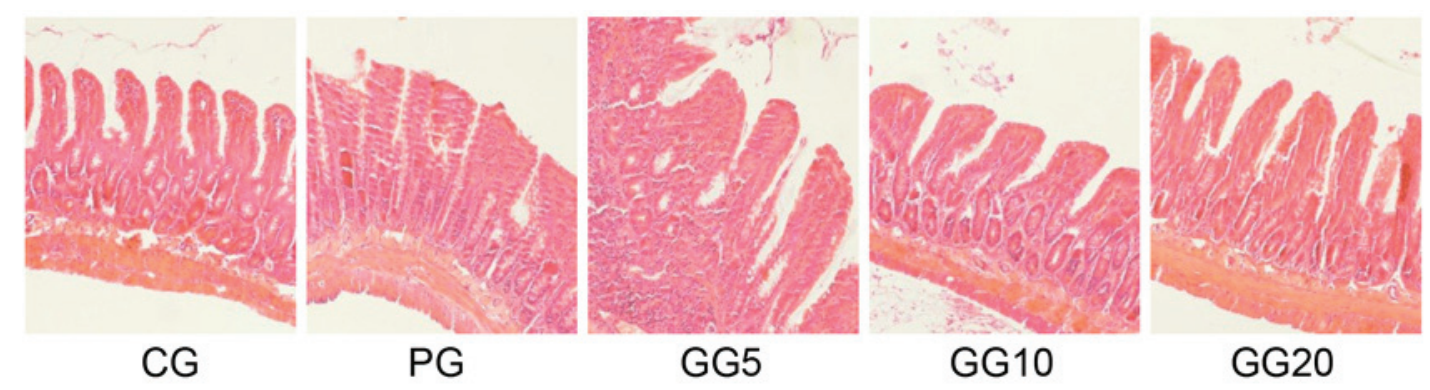

Figure 4. Histopathologic observation in the intestinal tissue assessed by hematoxylin and eosin staining (magnification, x100). GRb1, ginsenoside Rb1; CG, control group; PG, peritoneal air exposure for 3 h group; GG5, 5 mg/kg GRb1-treated PG group; GG10, 10 mg/kg GRb1-treated PG group; GG20, 20 mg/kg GRb1-treated PG group.

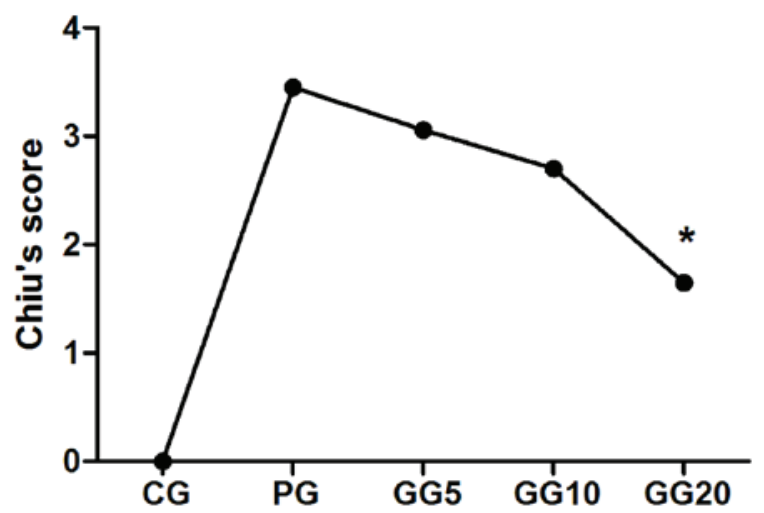

Figure 5. Effect of GRb1 on Chiu's scores. Results are presented as mean \pm standard deviation. ${ }^{*} \mathrm{P}<0.05$, vs. PG. GRb1, ginsenoside Rb1; CG, control group; PG, peritoneal air exposure for $3 \mathrm{~h}$ group; GG5, $5 \mathrm{mg} / \mathrm{kg}$ GRb1-treated PG group; GG10, 10 mg/kg GRb1-treated PG group; GG20, $20 \mathrm{mg} / \mathrm{kg}$ GRb1-treated PG group.

various pathological conditions, allowing FD4 to easily pass through the intestinal mucosal barrier. Therefore, the intestinal clearance of FD4 may also be chosen as a sensitive biomarker to detect intestinal mucosal barrier function $(19,26,27)$.

In the present study, a decrease was observed in serum D-LA and intestinal FD4 levels following GRb1 administration. Although there were no obvious structural differences detected via histopathological observation among the five groups, the Chiu's scores calculated for intestinal mucosal damage were decreased in the GG groups, compared with those in the PG group. Furthermore, this protective effect exerted by GRb1 occurred in a dose-dependent manner, with significant differences at 10 and $20 \mathrm{mg} / \mathrm{kg}$, respectively. These results demonstrate that GRb1 may be able to prevent intestinal mucosal barrier damage caused by peritoneal air exposure. Therefore, in clinical practice, perioperative treatment with GRb1 may be an effective method to prevent intestinal mucosal damage, ameliorate local and systemic inflammation, and further accelerate the course of convalescence in patients undergoing large abdominal surgery.

Notably, during the planning of the present study, it was unclear which pharmacological action exerted by GRb1 may have been responsible for these potentially protective effects, as GRb1 possesses various pharmacological activities including anti-oxidant action, anti-inflammation, and other protective effects on organ function $(5,28,29)$. However, it has been demonstrated in previous studies that intestinal inflammation has a role in the etiology of various gastrointestinal disorders, and reducing intestinal inflammation may relieve intestinal damage $(16,30,31)$. Therefore, the index of inflammation was measured via intestinal MPO activity. The results demonstrated that the activity of intestinal MPO, which is a good biomarker to assess inflammation condition (32), was increased in the PG group. These findings are in accordance with previous research (18). However, the activity of intestinal MPO was decreased by GRb1 administration in a dose-dependent manner, with significant differences detected at 10 and $20 \mathrm{mg} / \mathrm{kg}$. These results indicated that the anti-inflammatory action of GRb1 contributed to the protective effect on the intestinal mucosal barrier against damage following peritoneal air exposure. Further research is currently underway exploring other pharmacological activities of GRb1 and their potential protective roles in protecting the intestinal mucosal barrier following peritoneal air exposure.

In conclusion, the results of the present study demonstrated that GRb1 may be able to protect the intestinal mucosal barrier from damage induced by peritoneal air exposure, which may be associated with its anti-inflammatory action. GRb1 may be used as an effective perioperative treatment for intestinal mucosal barrier dysfunction in open abdominal surgery.

\section{Acknowledgements}

The authors would like to thank Dr Shanjun Tan of the Research Institute of General Surgery, Jinling Hospital, Nanjing University Medical School (Nanjing, China) for his excellent assistance in the design of this study and manuscript writing. The study was supported by the National Natural Science Foundation of China (grant no. 81171857), the Foundation of Key Supporting Discipline of Surgical Nutrition (grant no. 11-ZC24), and the Program of Medicine and Health Platform of Zhejiang, China (grant nos. 2011ZDA018 and 2013ADA014).

\section{References}

1. Tan SJ, Li N, Zhou F, Dong QT, Zhang XD, Chen BC and Yu Z: Ginsenoside Rb1 improves energy metabolism in the skeletal muscle of an animal model of postoperative fatigue syndrome. J Surg Res 191: 344-349, 2014.

2. Wang Y, Dong J, Liu P, Lau CW, Gao Z, Zhou D, Tang J, Ng CF and Huang Y: Ginsenoside Rb3 attenuates oxidative stress and preserves endothelial function in renal arteries from hypertensive rats. Br J Pharmacol 171: 3171-3181, 2014. 
3. Bi X, Xia X, Mou T, Jiang B, Fan D, Wang P, Liu Y, Hou Y and Zhao Y: Anti-tumor activity of three ginsenoside derivatives in lung cancer is associated with Wnt/ $\beta$-catenin signaling inhibition. Eur J Pharmacol 742: 145-152, 2014

4. Tan S, Yu W, Lin Z, Chen Q, Shi J, Dong Y, Duan K, Bai X, $\mathrm{Xu} \mathrm{L,} \mathrm{Li} \mathrm{J} \mathrm{and} \mathrm{Li} \mathrm{N:} \mathrm{Anti-inflammatory} \mathrm{Effect} \mathrm{of} \mathrm{ginsenoside} \mathrm{Rb1}$ contributes to the recovery of gastrointestinal motility in the rat model of postoperative ileus. Biol Pharm Bull 37: 1788-1794, 2014.

5. Tan S, Zhou F, Li N, Dong Q, Zhang X, Ye X, Guo J, Chen B and $\mathrm{Yu} \mathrm{Z}$ : Anti-fatigue effect of ginsenoside Rb1 on postoperative fatigue syndrome induced by major small intestinal resection in rat. Biol Pharm Bull 36: 1634-1639, 2013.

6. Zhang Y, Sun K, Liu YY, Zhang YP, Hu BH, Chang X, Yan L, Pan CS, Li Q, Fan JY, et al: Ginsenoside Rb1 ameliorates lipopolysaccharide-induced albumin leakage from rat mesenteric venules by intervening in both trans- and paracellular pathway. Am J Physiol Gastrointest Liver Physiol 306: G289-G300, 2014.

7. Wang J, Qiao L, Li S and Yang G: Protective effect of ginsenoside Rb1 against lung injury induced by intestinal ischemia-reperfusion in rats. Molecules 18: 1214-1226, 2013.

8. Choi JR, Hong SW, Kim Y, Jang SE, Kim NJ, Han MJ and Kim DH: Metabolic activities of ginseng and its constituents, ginsenoside rb1 and rg1, by human intestinal microflora. J Ginseng Res 35: 301-307, 2011.

9. Southall JC, Lee SW, Bessler M, Allendorf JD and Whelan RL: The effect of peritoneal air exposure on postoperative tumor growth Surg Endosc 12: 348-350, 1998.

10. Watson RW, Redmond HP, McCarthy J, Burke PE and Bouchier-Hayes D: Exposure of the peritoneal cavity to air regulates early inflammatory responses to surgery in a murine model. Br J Surg 82: 1060-1065, 1995.

11. Tan S, Yu W, Lin Z, Chen Q, Shi J, Dong Y, Duan K, Bai X, Xu L, Li J and Li N: Peritoneal air exposure elicits an intestinal inflammation resulting in postoperative ileus. Mediators Inflamm 2014: 924296, 2014

12. Gatt M, Reddy BS and MacFie J: Review article: Bacterial translocation in the critically ill-evidence and methods of prevention. Aliment Pharmacol Ther 25: 741-757, 2007.

13. Bao J, Tan S, Yu W, Lin Z, Dong Y, Chen Q, Shi J, Duan K, Bai X $\mathrm{Xu}$ L, et al: The effect of peritoneal air exposure on intestinal mucosal barrier. Gastroenterol Res Pract 2014: 674875, 2014.

14. Lau WL, Liu SM, Pahlevan S, Yuan J, Khazaeli M, Ni Z, Chan JY and Vaziri ND: Role of nrf2 dysfunction in uremia-associated intestinal inflammation and epithelial barrier disruption. Dig Dis Sci 60: 1215-1222, 2015.

15. Costes LM, van der Vliet J, van Bree SH, Boeckxstaens GE and Cailotto C: Endogenous vagal activation dampens intestinal inflammation independently of splenic innervation in postoperative ileus. Auton Neurosci 185: 76-82, 2014

16. Rychter $\mathrm{J}$ and Clavé $\mathrm{P}$ : Intestinal inflammation in postoperative ileus: Pathogenesis and therapeutic targets. Gut 62: 1534-1535, 2013

17. Vlug MS,Diepenhorst GM, van Koperen PJ, Renooij W, de Smet MB, Slors JF, Boermeester MA and Bemelman WA: Intestinal barrier function in patients undergoing colectomy. Colorectal Dis 13: 1432-1437, 2011.

18. Tan S, Yu W, Lin Z, Chen Q, Shi J, Dong Y, Duan K, Bai X, Xu L, Yu Z, et al: Berberine ameliorates intestinal mucosal barrier damage induced by peritoneal air exposure. Biol Pharm Bull 38: 122-126, 2015.
19. Yue C, Wang W, Tian WL, Huang Q, Zhao RS, Zhao YZ, Li QR and Li JS: Lipopolysaccharide-induced failure of the gut barrier is site-specific and inhibitable by growth hormone. Inflamm Res 62: 407-415, 2013.

20. Son JY, Chandler B, Feketova E, Qin Y, Quackenbush EJ and Deitch EA: Oral pretreatment with recombinant human lactoferrin limits trauma-hemorrhagic shock-induced gut injury and the biological activity of mesenteric lymph. J Surg Res 187: 270-277, 2014.

21. Bradford MM: A rapid and sensitive method for the quantitation of microgram quantities of protein utilizing the principle of protein-dye binding. Anal Biochem 72: 248-254, 1976.

22. Chiu CJ, McArdle AH, Brown R, Scott HJ and Gurd FN: Intestinal mucosal lesion in low-flow states. I. A morphological, hemodynamic and metabolic reappraisal. Arch Surg 101: 478-483, 1970.

23. Lu XG, Kang X, Zhou FQ, Wang XZ, Guo S, Fan ZW, Liang ZK and $\mathrm{Yu}$ J: Effects of pyruvate-enriched peritoneal dialysis solution on intestinal barrier in peritoneal resuscitation from hemorrhagic shock in rats. J Surg Res 193: 368-376, 2015.

24. Zhang W, Shen ZY, Song HL, Yang Y, Wu BJ, Fu NN and Liu T: Protective effect of bone marrow mesenchymal stem cells in intestinal barrier permeability after heterotopic intestinal transplantation. World J Gastroenterol 20: 7442-7451, 2014.

25. Wang H, Gong J, Wang W, Long Y, Fu X, Fu Y, Qian W and Hou X: Are there any different effects of Bifidobacterium, Lactobacillus and Streptococcus on intestinal sensation, barrier function and intestinal immunity in PI-IBS mouse model? PLoS One 9: e90153, 2014.

26. Wu BG, Peng TC, Tsai PS, Wang TY, Jeng MJ and Huang CJ: High-lipid enteral nutrition could partially mitigate inflammation but not lung injury in hemorrhagic shock rats. J Surg Res 184: 997-1005, 2013

27. Wattanasirichaigoon S, Menconi MJ, Delude RL and Fink MP: Effect of mesenteric ischemia and reperfusion or hemorrhagic shock on intestinal mucosal permeability and ATP content in rats. Shock 12: 127-133, 1999.

28. Wu Y, Yu Y, Szabo A, Han M and Huang XF: Central inflammation and leptin resistance are attenuated by ginsenoside $\mathrm{Rb} 1$ treatment in obese mice fed a high-fat diet. PLoS One 9: e92618, 2014.

29. Ni N, Liu Q, Ren H, Wu D, Luo C, Li P, Wan JB and Su H: Ginsenoside $\mathrm{Rb} 1$ protects rat neural progenitor cells against oxidative injury. Molecules 19: 3012-3024, 2014

30. Endo M, Hori M, Ozaki H, Oikawa T and Hanawa T: Daikenchuto, a traditional Japanese herbal medicine, ameliorates postoperative ileus by anti-inflammatory action through nicotinic acetylcholine receptors. J Gastroenterol 49: 1026-1039, 2014.

31. Karhausen J, Qing M, Gibson A, Moeser AJ, Griefingholt H, Hale LP, Abraham SN and Mackensen GB: Intestinal mast cells mediate gut injury and systemic inflammation in a rat model of deep hypothermic circulatory arrest. Crit Care Med 41: e200-e210, 2013.

32. Nussbaum C, Klinke A, Adam M, Baldus S and Sperandio M: Myeloperoxidase: A leukocyte-derived protagonist of inflammation and cardiovascular disease. Antioxid Redox Signal 18: 692-713, 2013. 